[1] E. Bombieri, Density theorems for the zeta function, 1969 Number Theory Institute (Proc. Sympose Pure Math., Vol. XX, State Univ. New York, Stony Brook, N. Y., 1969) pp. 352-358, Amer. Math. Soc., Providence, R. I., 1971.

[2] - A note on the large sieve, Acta Arith. 18 (1971), pp. 401-404.

[3] H. Daven port, Multiplicative number theory, Markham, Chicago 1967.

[4] W. J. Ellison and M. Mendès France, Les nombres premiers, Hermann, Paris 1975.

[5] G. Halâsz and P. Turán, On the distribution of roots of Riemann zeta and allied functions, I, J. Number Theory 1.(1969), pp. 121-137.

[6] - - On the distribution of roots of Riemann zeta and allied functions, $I I$, Acta Math. Acad. Sci. Hungar. 21 (1970), pp. 403-419.

[7] A. A. Karatsuba, Elements of analytic number theory (Russian), Nauka, Moscow 1975.

[8] H. L. Montgomery, Topics in multiplicative number theory, LNM 227, Springer, BerlinHeidelberg-New York 1971.

[9] J. Pintz, On Siege?'s theorem, Acta Arith. 24 (1974), pp. 543-551.

[10] - Elementary methods in the theory of L-functions IV, The Heilbronn phenomenom, ibid. 31 (1976), pp. 419-429.

[11] - Elementary methods in the theory of L-functions, VII, Real zeros of real L-functions, ibid. 33 (1977), pp. 89-98.

[12] H.-E. Richert, Zur Abschätzung der Riemannschen Zetafunktion in der Nähe der Vertikalen $\sigma=1$, Math. Ann. 169 (1967), pp. 97-101.

[13] E. C. Titchmarsh, The Theory of the Riemann Zeta-Function, Oxford University Press, Oxford 1948.

MATHEMATICAL INSTITUTE OF THE HUNGARIAN

ACADEMY OF SCIENCES

H-1053 Budapest, Reältanoda v. 13-15

Received on 28.5 .1986

\section{Nombres hautement composés}

par

JEAN-LOUIS Nicolas (Limoges)

A Paul Erdös

pour fêter son 75ème anniversaire

1. Introduction. Dans un long mémoire paru en 1915 , S. Ramanujan a défini et étudié les nombres hautement composés, c'est-à-dire les nombres qui ont strictement plus de diviseurs que les nombres qui les précèdent (cf. [14], $\mathrm{n}^{\circ} 15$ ). Appelons $Q(X)$ la quantité de nombres hautement composés inférieurs ou égaux à $X$. S. Ramanujan a en particulier démontré que

$$
\lim _{X \rightarrow+\infty} Q(X) / \log X=+\infty
$$

mais n'a pas donné de majoration pour $Q(X)$. C'est pourtant un sujet qui l'intéressait puisque dans l'article écrit avec G. H. Hardy: Asymptotic formulae for the distribution of integers of various types (cf. [14], $\mathrm{n}^{\circ} 34$ ), il étudie les nombres de la forme:

$$
2^{a_{1}} 3^{a_{2}} \ldots p_{k}^{a_{k}}
$$

où $p_{k}$ désigne le $k^{\text {ième }}$ nombre premier, et $a_{1} \geqslant a_{2} \geqslant \ldots \geqslant a_{k} ;$ parmi les nombres de cette forme figurent les nombres hautement composés.

En 1944, P. Erdős démontre (cf. [3]) qu'il existe $c^{\prime}>0$ tel que

$$
Q(X) \geqslant(\log X)^{1+c^{\prime}}
$$

pour $X$ assez grand.

Un des outils essentiels dans sa démonstration, est le résultat de Hoheisel:

$$
\begin{aligned}
\text { LeMme 1. Soit } \pi(x) & =\sum_{p \leqslant x} 1 \text {. Il existe } \tau<1 \text { tel que } \\
& \pi\left(x+x^{\tau}\right)-\pi(x) \sim x^{\tau} / \log x
\end{aligned}
$$

Nous désignerons par $\tau$ un nombre réel $<1$ pour lequel on a, pour tout $x$ assez grand:

$$
\pi\left(x+x^{\tau}\right)-\pi(x) \gg x^{\tau} / \log x
$$


D'après D. R. Heath-Brown et $\mathrm{H}$. Iwaniec, on peut prendre pour $\tau$ n'importe quel nombre $>11 / 20$ (cf. [5]).

En 1971, j'ai donné dans [9] la majoration:

$$
Q(X)=O(\log X)^{c^{\prime \prime}} \text {. }
$$

Un autre outil essentiel introduit dans la démonstration de ce résultat était les approximations diophantiennes du nombre

$$
\theta=\log (3 / 2) / \log 2=0.5849625 \ldots
$$

A la suite des travaux de Baker sur les formes linéaires de logarithmes de nombres algébriques, Feldman démontrait l'existence d'une constante $x$ telle que

$$
|a \theta-b| \gg|a|^{-x}
$$

pour tous $a$ et $b$ entiers, $a \neq 0$.

On verra ci-dessous dans le lemme 2 des valeurs explicites de cette constante $\varkappa$, obtenues plus récemment, et qui vont nous permettre de donner une valeur précise de $c^{\prime \prime}$.

Les nombres hautement composés ont été généralisés à d'autres fonctions arithmétiques:

$d_{t}(n)=$ nombre de façons de décomposer $n$ en $t$ facteurs; $t=2$ correspond aux nombres hautement composés de Ramanujan (cf. [13])

$\sigma(n)=$ somme des diviseurs de $n$, cf. [1], [12], [4], [16]

$\varphi(n)=$ fonction d'Euler, cf. [12], [6]

$a(n)=$ nombres de groupes abéliens d'ordre $n, \mathrm{cf}$. [11]

Une table des 5000 premiers nombres hautement composés de Ramanujan a été obtenue par G. Robin et $\mathrm{H}$. te Riele et $\mathrm{G}$. Robin a utilisé la méthode des "bénéfices" décrite au $\S 4$, pour calculer les nombres hautement composés voisins d'un nombre fixé (cf. [15]).

Dans [10], j'ai étudié les nombres largement composés, c'est-à-dire les nombres $n$ tels que

$$
m \leqslant n \Rightarrow d(m) \leqslant d(n)
$$

où $d(n)$ désigne le nombre de diviseurs de $n$. Ces nombres sont beaucoup plus nombreux que les nombres hautement composés.

J'ai très souvent parlé avec Paul Erdõs au sujet des nombres hautement composés, notamment pendant la rédaction de l'article [4]. Le théorème 2 cidessous et ses corollaires apportent une réponse à des questions qu'il m'avait posées il y a quelques mois.

\section{Résultats.}

THÉORÈME 1. Soit $Q(X)$ le nombre de nombres hautement composés de Ramanujan $\leqslant X$. On pose $\theta=(\log (3 / 2)) / \log 2$. On définit $\tau$ et $\varkappa$ par (1) et (2). On définit $c(X)$ par:

$$
\begin{gathered}
Q(X)=(\log X)^{c(X)}, \\
c_{1}=\limsup c(X) \quad \text { et } \quad c_{2}=\liminf c(X) .
\end{gathered}
$$

(i) On a $c_{1}<1+g(\gamma)$, où $\gamma$ est défini par (19) et $g$ par (14). La valeur de $x$ donnée par $M$. Waldschmidt (cf. lemme 2) donne $c_{1} \leqslant 3.48$. La valeur de $\chi$ annoncée par $G$. Rhin donne $c_{1} \leqslant 1.82$.

(ii) On $a c_{2} \leqslant 1.44$.

Remarque. Il est démontré dans [9] que $c_{2} \geqslant\left(1+\frac{1-\tau}{3}\right) \frac{\log (15 / 8)}{\log 2}$ $\geqslant 1.136$ et conjecturé que $c_{1}=c_{2}=\log 30 / \log 16=1.227$.

THÉORÈmE 2. Soit $n_{j}^{\prime}$ la suite des nombres largement composés, et soit $n_{j}$ la suite des nombres hautement composés de Ramanujan. Il existe des nombres $>0, \alpha_{1}, \alpha_{2}, \alpha_{3}, \alpha_{4}$ tels que l'on ait, pour $j$ assez grand:

$$
\frac{n_{j+1}^{\prime}}{n_{j}^{\prime}} \geqslant 1+\frac{1}{\exp \left(\left(\log n_{j}^{\prime}\right)^{\alpha_{1}}\right)},
$$

(iv) si $n_{j}<n<n_{j+1}$ et $d(n)<d\left(n_{j}\right)$ alors:

$$
\frac{d(n)}{d\left(n_{j}\right)} \leqslant 1-\frac{1}{\exp \left(\left(\log n_{j}\right)^{\alpha_{4}}\right)}
$$

Corollarre 1. Soit $f(n)$ une fonction croissante vérifiant

$$
\limsup \frac{f(n) \log \log n}{\log n}<\log 2 \text {. }
$$

On considère les nombres $n$ qui sont $(d+f)$ hautement composés, c'est-à-dire tels que

$$
m<n \Rightarrow d(m)+f(m)<d(n)+f(n) .
$$

Alors l'ensemble des nombres $(d+f)$ hautement composés est égal à l'ensemble des nombres largement composés augmenté au plus d'un ensemble fini.

De même l'ensemble des nombres $(d-f)$ hautement composés est égal à l'ensemble des nombres hautement composés de Ramanujan diminué d'au plus un ensemble fini. 
Corollaire 2. A un nombre fini d'exceptions près, les nombres n qui sont tels que

$$
m<n \Rightarrow d(m)+d(m+1)<d(n)+d(n+1)
$$

vérifient: $n$ ou $n+1$ est largement composé.

Sauf peut-être un nombre fini, les nombres hautement composés de Ramanujan $\left(n_{j}\right)$ vérifient:

$$
m<n_{j} \Rightarrow d(m-1)+d(m)<d\left(n_{j}-1\right)+d\left(n_{j}\right) .
$$

\section{Quelques lemmes.}

Lemme 2 (M. Waldschmidt). Pour tout $a, b \in N, a \neq 0$, on $a$ :

$$
|a \log (3 / 2)-b \log 2| \geqslant A a^{-x}
$$

avec $x=2^{49} \log 3$ et $A=\exp (-x(1+\log \log 3))$

Démonstration. Si $b \geqslant a$, on a:

$$
b \log 2-a \log (3 / 2) \geqslant a / 4 \geqslant A a^{-x} \text {. }
$$

On peut donc supposer $b \leqslant a$, et l'on applique la proposition $3.8 \mathrm{de} \mathrm{M}$. Waldschmidt (cf. [20]), avec $K=Q, n=2, \alpha_{1}=2, \alpha_{2}=3 / 2, \beta_{0}=0, \beta_{1}=$ $-b, \beta_{2}=a, V_{1}=1, V_{2}=\log 3, W=\log a, E=e, V_{1}^{+}=1, V_{2}^{+}=\log 3, q=2$. $\mathrm{La}$ constante énoncée dans la proposition 3.8 est $2^{50}$, mais dans la démonstration, il est indiqué que l'on peut prendre:

$$
c_{1}(2, q)=2^{41} q^{8}=2^{49} \text {. }
$$

Remarque. G. Rhin vient d'annoncer qu'il pouvait démontrer le lemme 2 avec $\varkappa=14$, et une valeur de $A$ non encore explicitée.

LEMME 3 (Théorème des 3 distances de V. Sós et S. Świerczkowski). Soit $\theta \notin Q, m^{\prime}, m^{\prime \prime} \in Z, m^{\prime}<m^{\prime \prime}, m=m^{\prime \prime}-m^{\prime},\{x\}=$ partie fractionnaire de $x$. On ordonne les nombres $\{i \theta\}, m^{\prime} \leqslant i \leqslant m^{\prime \prime}$ en une suite ordonnée $t_{0}<t_{1}<\ldots<t_{m}$. Alors les différences $t_{j+1}-t_{j}$ prennent au plus 3 valeurs. Soit $u_{k} / v_{k}$ les convergents successifs de $\theta$. On détermine $j$ par: $v_{j} \leqslant m<v_{j+1}$. On pose

$$
r=\left[\frac{m-v_{j-1}}{v_{j}}\right] \quad \text { et } \quad v=r v_{j}+v_{j-1}
$$

Alors ces trois différences sont $\left\|v_{j} \theta\right\|,\|v \theta\|,\|v \theta\|+\left\|v_{j} \theta\right\|$, où $\|x\|=\min |x-n|$.

La démonstration de ce lemme se trouve dans [18] qui donne un historique complet du sujet; cf. aussi [2].

LEMME 4. Soit $\sigma_{k} \in\{-1,0,+1\}$. On peut écrire le produit:

$$
\prod_{1 \leqslant k \leqslant y}\left(\frac{k+1}{k}\right)^{\sigma_{k}}=\frac{A}{B}
$$

avec: $\log \max (A, B) \leqslant \frac{2}{3} y \log y(1+o(1))$
Démonstration. On a évidemment la majoration

$$
\max (A, B) \leqslant(y+1) ! \text {. }
$$

On observe que:

$$
\left(\frac{k+1}{k}\right)^{\sigma_{k}}\left(\frac{2 k+1}{2 k}\right)^{a_{2 k}}\left(\frac{2 k+2}{2 k+1}\right)^{\sigma_{2 k+1}}=\frac{a}{b}
$$

où $\max (a, b) \leqslant(2 k+2)^{2}$

En effet, si l'un des trois exposants $\sigma$ est nul, c'est clair. Si $\sigma_{2 k}$ et $\sigma_{2 k+1}$ ont même signe, il y a simplification par $2 k+1$; sinon $2 k(2 k+2)$ se met en facteur dans le produit des deux derniers facteurs, et il y a simplification avec le premier facteur.

Il s'ensuit que pour $u \geqslant 1$,

$$
\prod_{u \leqslant k \leqslant 4 u-1}\left(\frac{k+1}{k}\right)^{\sigma_{k}}=\frac{a_{u}}{b_{u}}
$$

avec $\max \left(a_{u}, b_{u}\right) \leqslant(4 u)^{2 u}$. Il suffit d'appliquer la formule précédente pour $u \leqslant k \leqslant 2 u-1$

Enfin, soit $t$ entier défini par:

$$
4^{t} \leqslant y<4^{t+1} \text {. }
$$

On considère les intervalles $\left(u_{j}, 4 u_{j}-1\right)$ avec $u_{j}=\left[y / 4^{j}\right]$ pour $j=1, \ldots, t$. La réunion de ces intervalles recouvre l'intervalle $(1, y)$, sauf au plus $4(t+1)$ nombres. On a donc:

$$
\max (A, B) \leqslant\left(\prod_{1 \leqslant j \leqslant t}\left(4 u_{j}\right)^{2 u_{j}}\right)(y+1)^{4(t+1)}
$$

et

$$
\log \max (A, B) \leqslant \sum_{j=1}^{t} 2 u_{j} \log (4 y)+O(\log y)^{2} \leqslant \frac{2}{3} y \log y(1+o(1))
$$

Michel Langevin a observé que, lorsque $y$ est pair, la quantité:

$$
\frac{1}{2} \frac{3}{2} \frac{3}{4} \frac{5}{4} \ldots \frac{y-1}{y} \frac{y+1}{y}
$$

vaut $\frac{y+1}{4^{y}}\left(\begin{array}{c}y \\ y / 2\end{array}\right)^{2}$ et est de la forme $A / 2^{b}$ avec $A$ impair, et $4^{y} / y \leqslant 2^{b} \leqslant 4^{y}$. On en déduit que, dans ce cas,

$$
\log \max (A, B) \geqslant y(\log 4)(1+o(1)) .
$$

4. Etude des nombres de petit bénéfice. On dit que $N$ est hautement composé supérieur (h.c.s) s'il existe $\varepsilon>0$, tel que, pour tout $M$ entier, on ait: 
Rappelons les propriétés de ces nombres (cf. [14], p. 111 et [9]): Etant donné $\varepsilon, 0<\varepsilon<1$, il existe un nombre h.c.s. associé à $\varepsilon$ dont la décomposition en facteurs premiers

$$
N=\prod_{\lambda} \lambda^{a} \lambda
$$

est donnée par:

$$
a_{\lambda}=\left[\frac{1}{\lambda^{2}-1}\right]
$$

où $[x]$ désigne la partie entière de $x$.

On attache à $N=N_{\varepsilon}$ les nombres:

$$
x=2^{1 / s} \quad \text { et } \quad x_{k}=x^{\log (1+1 / k) \log 2} .
$$

On a alors:

$$
a_{\lambda}=k \Leftrightarrow x_{k+1}<\lambda \leqslant x_{k}
$$

et:

$$
x \sim \log N .
$$

Soit $p \leqslant x<P$ les nombres premiers encadrant $x$. Le plus grand nombre premier qui divise $N$ est $p$, et le nombre hautement composé supérieur $N^{\prime}$ suivant $N$ vérifie $N^{\prime} \leqslant N P$ et $d\left(N^{\prime}\right) \leqslant 2 d(N)$.

$N$ et $\varepsilon$ étant fixés, on définit pour $M \in N$ le "bénéfice" de $M$ :

$$
\text { bén } M=\varepsilon \log \frac{M}{N}-\log \frac{d(M)}{d(N)} \text {. }
$$

D'après (4) on a toujours bén $M \geqslant 0$.

Soit $\varrho>0$. On définit

$$
\begin{gathered}
k^{\prime}(\varrho)=\left[\frac{1}{e^{e^{\operatorname{jog} 2}-1}}\right], \\
k^{\prime \prime}=1+\left[\frac{\log x}{(\log \log x-\log \log \log x) \log 2}\right] .
\end{gathered}
$$

On observe que:

$$
x_{k^{\prime \prime}} \leqslant x^{1 / k^{\prime} \log 2} \leqslant \log x / \log \log x .
$$

Soit maintenant $M$ un nombre quelconque vérifiant:

$$
d(N) \leqslant d(M)<2 d(N)
$$

$$
\text { bén } M \leqslant x^{-\varrho} \text {. }
$$

- On écrit la décomposition en facteurs premiers de $M$ :

$$
M=\prod \lambda^{b_{\lambda}}
$$

On a d'abord, dès que $N$ est assez grand:

$$
\left|b_{\lambda}-a_{\lambda}\right| \leqslant 1 \text {. }
$$

En effet, la démonstration de la proposition 5 de [9] montre que si l'on avait $\left|b_{\lambda}-a_{\lambda}\right| \geqslant 2$, cela entrainerait

$$
\text { bén } M \gtrsim \frac{(\log 2)^{4}}{(\log x)^{2}}>x^{-e} .
$$

On a ensuite:

$$
\text { bén } M=\sum_{\lambda}\left(\varepsilon\left(b_{\lambda}-a_{\lambda}\right) \log \lambda-\log \frac{b_{\lambda}+1}{a_{\lambda}+1}\right)
$$

et l'on observe que, à cause du choix de $a_{\lambda}$, chacun des termes de la sommation est $\geqslant 0$.

En particulier si $x_{k}<\lambda \leqslant x_{k-1}$, et si $b_{\lambda}=a_{\lambda}+1$, on a:

$$
\text { bén } M \geqslant \operatorname{bén}(\lambda N)=\varepsilon \log \left(\lambda / x_{k}\right) \text {. }
$$

De même, si $x_{k+1}<\lambda \leqslant x_{k}$ et si $b_{\lambda}=a_{\lambda}-1$, on a:

bén $M \geqslant \operatorname{bén}(N / \lambda)=\varepsilon \log \left(x_{k} / \lambda\right)$.

La condition (9) entraîne dans le premier cas

$$
\lambda / x_{k} \leqslant \exp \left(x^{-\ell} \log x / \log 2\right)
$$

soit, pour $x$ assez grand,

$$
\frac{\lambda-x_{k}}{x_{k}} \leqslant 2 \frac{x^{-\varrho} \log x}{\log 2}
$$

et encore

$$
\lambda-x_{k} \leqslant \frac{2}{\log 2} x^{(\log (1+1 / k) / \log 2)-e} \log x .
$$

Dans le deuxième cas on obtiendrait de même:

$$
x_{k}-\lambda \leqslant \frac{2}{\log 2} x^{(\log (1+1 / k) / \log 2)-a} \log x .
$$

Supposons que $k>k^{\prime}(\varrho)$, alors le deuxième membre de (11) et (12) tend vers 0 , et il ne peut donc n'y avoir qu'un seul nombre premier très voisin de $x_{k}$ dont l'exposant puisse varier.

Supposons maintenant $k \leqslant k^{\prime}(\varrho)$. Les relations (11) et (12) montrent que 
$\lambda \sim x_{k}$. Supposons qu'il y ait $n$ nombres premiers $x_{k}<\lambda_{1}<\lambda_{2}<\ldots$ $\ldots<\lambda_{n} \leqslant x_{k-1}$ tels que $b_{\lambda_{i}}=a_{\lambda_{i}}+1$. On aurait alors:

$$
\text { bén } M \geqslant \sum_{i=1}^{n} \varepsilon \log \left(\lambda_{i} / x_{k}\right) \geqslant \varepsilon \sum_{i=1}^{n} \frac{\lambda_{i}-x_{k}}{\lambda_{n}} \geqslant \frac{\varepsilon}{\lambda_{n}} \sum_{i=1}^{n} 2(i-1) \text {. }
$$

Comme $\lambda_{n} \sim x_{k}$, l'inégalité (9) entraîne:

$$
n \lesssim \sqrt{\varepsilon^{-1} x_{k} x^{-e}}
$$

On pourrait obtenir une majoration un peu meilleure, en minorant $\lambda_{i}-x_{k}$ par $(i \log i) / 2$, à l'aide de l'inégalité de Brun-Titchmarsh (cf. [7]):

$$
\pi(x+y)-\pi(x) \leqslant 2 y / \log y
$$

valable pour tout $x>0$ et $y>1$, mais nous n'en aurons pas besoin ici.

On raisonnerait de même pour $n$ nombres premiers $\lambda_{i}$ compris entre $x_{k+1}$ et $x_{k}$ et tels que $b_{\lambda_{i}}=a_{\lambda_{i}}-1$.

Pour $2 \leqslant k \leqslant k^{\prime}(\varrho)$, on définit:

$$
v_{k}=2 \sqrt{x_{k} x^{-\ell} \log x}>\sqrt{\varepsilon^{-1} x_{k} x^{-\ell}} .
$$

On peut donc associer à $M$ les paramètres suivants:

Pour les nombres premiers $\lambda \leqslant x_{k^{\prime \prime}}, b_{\lambda}$ avec $\left|b_{\lambda}-a_{\lambda}\right| \leqslant 1$.

Pour les indices $k, k^{\prime}<k \leqslant k^{\prime \prime}, \sigma_{k} \in\{-1,0,1\}$.

Pour ces valeurs de $k$, il y a au plus un nombre premier $\lambda \in\left[x_{k}-1, x_{k}\right.$ $+1]$ avec $b_{\lambda} \neq a_{\lambda}$. S'il n'y en a pas, on pose $\sigma_{k}=0$. S'il y en a un tel que:

$$
\lambda \geqslant x_{k} \text { et } b_{\lambda}=a_{\lambda}+1, \quad \text { on pose } \sigma_{k}=+1
$$

sil en a un tel que:

$$
\lambda \leqslant x_{k} \text { et } b_{\lambda}=a_{\lambda}-1, \quad \text { on pose } \sigma_{k}=-1 \text {. }
$$

Lorsque $k=k^{\prime \prime}, \sigma_{k}$ vaut soit 0 , soit +1 .

Pour les indices $k, 1 \leqslant k \leqslant k^{\prime}$ :

$\mu_{k}^{\prime}$. désigne le nombre de nombres premiers $\lambda \in\left[x_{k}, x_{k-1}\right]$ tels que $b_{\lambda}=a_{\lambda}+1$;

$\mu_{k}^{\prime \prime}$ désigne le nombre de nombres premiers $\lambda \in\left[x_{k+1}, x_{k}\right]$ tels que $b_{\lambda}=a_{\lambda}-1$.

Avec ces paramètres, on a:

$$
\frac{d(M)}{d(N)}=\prod_{\lambda \leqslant x_{k^{\prime \prime}}} \frac{b_{\lambda}+1}{a_{\lambda}+1} \prod_{k^{\prime}<k \leqslant k^{\prime \prime}}\left(\frac{k+1}{k}\right)^{\sigma_{k}} \prod_{1 \leqslant k \leqslant k^{\prime}}\left(\frac{k+1}{k}\right)^{\mu_{k}^{\prime}-\mu_{k}^{\prime \prime}}
$$

Propostrion 1. Le nombre de valeurs possibles pour $d(M)$ lorsque $d(N) \leqslant d(M)<2 d(N)$ et bén $M \leqslant x^{-e}$ est $\leqslant(\log N)^{g(\ell)+o(1)} ;$ le o(1) est uniforme pour $\varrho \geqslant \varrho_{0}>0$, et $g$ est défini par:

$$
g(\varrho)=\sum_{p=3}^{k^{\prime}+1} \frac{1}{2}\left(\frac{\log (p /(p-1))}{\log 2}-\varrho\right)
$$

où $k^{\prime}$ est défini par (6). Lorsque $\varrho \geqslant \theta, g(\varrho)=0$.

Démonstration. Le nombre de valeurs possibles pour le premier produit de (13) est

$$
3^{\pi\left(x_{k^{\prime \prime}}\right)} \leqslant 3^{\pi(\log x)}=x^{o(1)}=(\log N)^{o(1)}
$$

Le nombre de valeurs possibles pour le deuxième produit est inférieur à $3^{k^{\prime \prime}}=(\log N)^{o(1)}$.

Pour le troisième produit, on pose $\mu_{k}=\mu_{k}^{\prime}-\mu_{k}^{\prime \prime}$ et on écrit la décomposition en facteurs premiers:

$$
\prod_{2 \leqslant k \leqslant k^{\prime}}\left(\frac{k+1}{k}\right)^{\mu_{k}}=\prod_{2 \leqslant p \leqslant k^{\prime}+1} p^{\alpha_{p}}, \quad \alpha_{p} \in Z .
$$

Appelons $v_{p}(k)$ l'exposant de la plus grande puissance de $p$ divisant $k$. On a:

$$
\begin{aligned}
\alpha_{p} & =\sum_{k=1}^{k^{\prime}} \mu_{k} v_{p}(k+1)-\mu_{k} v_{p}(k)=\sum_{k=2}^{k^{\prime}} v_{p}(k)\left(\mu_{k-1}-\mu_{k}\right)+\mu_{k^{\prime}} v_{p}\left(k^{\prime}+1\right) \\
& =\mu_{p-1}-\mu_{p}+\sum_{k=2 p}^{k^{\prime}} v_{p}(k)\left(\mu_{k-1}-\mu_{k}\right)+\mu_{k^{\prime}} v_{p}\left(k^{\prime}+1\right)=\mu_{p-1}(1+o(1))
\end{aligned}
$$

où le petit $o$ dépend de $\varrho_{0}$. En effet, on a:

$$
\left|\mu_{k}\right| \leqslant v_{k}
$$

et pour $1 \leqslant k \leqslant k^{\prime}, v_{k}=o\left(v_{k-1}\right)$.

Lorsque tous les autres paramètres ont été choisis dans (13), le choix de $\alpha_{2}$ est imposé par la condition $d(N) \leqslant d(M)<2 d(N)$. Pour chaque $\alpha_{p}$ on a donc au plus $2 v_{p-1}$ choix, et l'on a:

$$
\prod_{p=3}^{k^{\prime}+1}\left(2 v_{p-1}\right)=x^{g(Q)+o(1)}
$$

Proposition 2. Soit $M$ et $M^{\prime}$ deux nombres vérifiant

$$
\begin{gathered}
d(N) \leqslant d\left(M^{\prime}\right)<d(M)<2 d(N), \\
\varrho<\theta, \quad \text { bén } M \leqslant x^{-e} \quad \text { et } \quad \text { bén } M^{\prime} \leqslant x^{-\ell} .
\end{gathered}
$$

Alors, lorsque $N$ est assez grand, on $a$ :

$$
\frac{d(M)}{d\left(M^{\prime}\right)} \geqslant 1+\exp \left(-x^{(\theta-e) / 2+o(1)}\right)
$$


Démonstration. Observons d'abord que dans la formule (13) $\mu_{1}=\mu_{1}^{\prime}-\mu_{1}^{\prime \prime}$ ne peut pas être trop grand. En effet:

$$
\log \frac{d(M)}{d(N)}=\mu_{1} \log 2+R
$$

avec:

$$
R=\sum_{\lambda \leqslant x_{k^{\prime \prime}}} \log \frac{b_{\lambda}+1}{a_{\lambda}+1}+\sum_{k^{\prime}<k \leqslant k^{\prime \prime}} \sigma_{k} \log \frac{k+1}{k}+\sum_{2 \leqslant k \leqslant k^{\prime}} \mu_{k} \log \frac{k+1}{k}
$$

et

$$
|R| \leqslant\left(\pi\left(x_{k^{\prime \prime}}\right)+k^{\prime \prime}+\sum_{2 \leqslant k \leqslant k^{\prime}} v_{k}\right) \log 2=v_{2}(\log 2)(1+o(1))
$$

et comme $1 \leqslant d(M) / d(N)<2$, cela entraîne

(16)

$$
\left|\mu_{1}\right| \leqslant v_{2}(1+o(1)) \text {. }
$$

Ensuite, on écrit $d(M) / d\left(M^{\prime}\right)=A / B$, où $A$ et $B$ sont entiers, et l'on a:

D'après la définition de $a_{\lambda}$, on a:

$$
B \leqslant A \leqslant\left(a_{2}+2\right)^{\pi\left(x_{k^{\prime \prime}}\right)} \prod_{k^{\prime}<k \leqslant k^{\prime \prime}}(k+1)^{2}\left(\prod_{2 \leqslant k \leqslant k^{\prime}}(k+1)^{2 v_{k}}\right) 2^{2 v_{2}(1+o(1))}
$$

$$
a_{2} \leqslant \frac{1}{\varepsilon \log 2}=\frac{\log x}{(\log 2)^{2}}
$$

et l'on en déduit que:

$$
\log A \leqslant 2 v_{2}(\log 6)(1+o(1)) \leqslant x^{(\theta-\varrho) / 2+o(1)} .
$$

Proposition 3. Soit $M$ et $M^{\prime}$ deux nombres vérifiant $M^{\prime}<M$,

$$
\begin{array}{cl}
d(N) \leqslant d(M)<2 d(N), & d(N) \leqslant d\left(M^{\prime}\right)<2 d(N), \\
\text { bén } M \leqslant x^{-\varrho}, & \text { bén } M^{\prime} \leqslant x^{-} \varrho
\end{array}
$$

Alors on $a$ :

$$
M / M^{\prime} \geqslant 1+\exp \left(-(\log N)^{1-\varrho+o(1)}\right)
$$

Démonstration. D'après ce qui précède, les deux nombres $M$ et $M^{\prime}$ ont en commun de nombreux facteurs premiers, et l'on peut écrire

$$
M / M^{\prime}=A / B
$$

avec

$$
B<A \leqslant \prod_{p \leqslant x_{k^{\prime \prime}}} p^{2} \prod_{k^{\prime}<k \leqslant k^{\prime \prime}}\left(x_{k}+1\right) \prod_{1 \leqslant k \leqslant k^{\prime}}\left(2 x_{k}\right)^{t_{k}}
$$

où $t_{k}=\max \left[\mu_{k}^{\prime}(M)+\mu_{k}^{\prime \prime}\left(M^{\prime}\right), \mu_{k}^{\prime \prime}(M)+\mu_{k}^{\prime}\left(M^{\prime}\right)\right]$.
En utilisant $\mu_{k} \leqslant v_{k}$, on obtient comme dans la proposition 2:

$$
\log A \leqslant 2 v_{1} \log (2 x)(1+o(1)) .
$$

Proposition 4. Soit $N$ un nombre hautement composé supérieur assez grand, $n$ le nombre hautement composé suivant $N$, on a, pour $N$ assez grand:

$$
\frac{n}{N} \geqslant 1+\frac{1}{(\log N)^{0.9618}}
$$

Démonstration. On choisit $\varrho=0.96$. Si l'on a bén $n \geqslant x^{-e}$, cela entraîne:

$$
\varepsilon \log \frac{n}{N} \geqslant x^{-e}+\log \frac{d(n)}{d(N)}>x^{-e}
$$

et donc

$$
n / N \geqslant 1+x^{-\varrho+o(1)}=1+(\log N)^{-e+o(1)} .
$$

Si l'on a bén $n \leqslant x^{-e}$, par (6) on a $k^{\prime}=1$ et la formule (13) donne:

$$
\frac{d(n)}{d(N)}=\prod_{\lambda \leqslant x_{k^{\prime \prime}}} \frac{b_{\lambda}+1}{a_{\lambda}+1} \prod_{2 \leqslant k \leqslant k^{\prime \prime}}\left(\frac{k+1}{k}\right)^{\sigma_{k}} 2^{\mu_{1}}
$$

D'après le lemme 4 , le deuxième produit s'écrit $A^{\prime \prime} / B^{\prime \prime}$ avec

$$
\log \left(\max \left(A^{\prime \prime}, B^{\prime \prime}\right)\right) \leqslant \frac{2}{3} k^{\prime \prime} \log k^{\prime \prime}(1+o(1)) \leqslant 0.9618 \log x .
$$

Le premier produit s'écrit $A^{\prime} / B^{\prime}$ avec:

$$
\log \max \left(A^{\prime}, B^{\prime}\right) \leqslant \pi\left(x_{k^{\prime \prime}}\right) \log \left(a_{2}+2\right)=o(\log x)
$$

en utilisant (8) et (17).

Ensuite $\mu_{1}$ ne peut pas être trop grand. En effet, on a:

$$
0<\log \frac{d(n)}{d(N)}=\mu_{1} \log 2+R \leqslant \log 2
$$

avec

$$
\begin{aligned}
|R| & \leqslant \sum_{\lambda \leqslant x_{k^{\prime \prime}}}\left|\log \frac{a_{\lambda}}{a_{\lambda}+1}\right|+\sum_{2 \leqslant k \leqslant k^{\prime \prime}} \log \frac{k+1}{k} \leqslant \sum_{\lambda \leqslant x_{k^{\prime \prime}}} \frac{1}{a_{\lambda}}+\log \frac{k^{\prime \prime}+1}{2} \\
& \leqslant(1+o(1)) \frac{\log 2 \log x_{k^{\prime \prime}}}{\log x} \pi\left(x_{k^{\prime \prime}}\right)+\log k^{\prime \prime} \leqslant \log k^{\prime \prime}+o(1)
\end{aligned}
$$

Cela entraîne:

$$
\left|\mu_{1}\right| \leqslant \frac{\log k^{\prime \prime}}{\log 2}+O(1) \quad \text { et } \quad 2^{\left|\mu_{1}\right|}=O\left(k^{\prime \prime}\right)=o(\log x)
$$


On obtient finalement:

$$
\frac{d(n)}{d(N)}=\frac{A}{B}=\frac{A^{\prime}}{B^{\prime}} \frac{A^{\prime \prime}}{B^{\prime \prime}} 2^{\mu_{1}}
$$

ce qui entraîne:

$$
\log (\max (A, B)) \leqslant 0.9618(\log x)
$$

et

$$
\frac{A}{B} \geqslant \frac{B+1}{B} \geqslant 1+\frac{1}{x^{0.9618}}
$$

Cette proposition améliore le lemme 1 de [10] en ce sens qu'elle est valable pour tout $N$ h.c.s. Mais l'exposant 0.9618 est moins bon. Il pourrait être diminué par une meilleure estimation des produits $\prod(k+1 / k)^{\sigma}$.

5. Démonstration du théorème 1. Nous utilisons le résultat du théorème 1 de [9]: Si $M$ est hautement composé, si $N$ est le nombre hautement composé supérieur précédant $M$, et associé à $\varepsilon$, si $x=2^{1 / \varepsilon}$, alors:

$$
\text { bén } M=O\left(x^{-\gamma}\right)
$$

avec

$$
\gamma=\theta(1-\tau) /(x+1)
$$

Comme il y a au plus $O(\log X / \log \log X)$ nombres h.c.s. $\leqslant X$, la proposition 1 nous donnent:

$$
Q(X) \leqslant(\log X)^{1+g(y)+o(1)} .
$$

B. Rosser et L. Schoenfeld donne (cf. [17]):

$$
\prod_{p=2}^{x} \frac{p}{p-1} \leqslant e^{c} \log x\left(1+\frac{1}{\log ^{2} x}\right) \quad(x \geqslant 1)
$$

(où $C=0.577 \ldots$ est la constante d'Euler) et

$$
\pi(x) \geqslant x / \log x \quad(x \geqslant 17) .
$$

Il s'ensuit que

$$
\sum_{p=3}^{x} \log \frac{p}{p-1} \leqslant C+\log \log x+\frac{1}{\log ^{2} x}-\log 2
$$

et que, pour $\varrho \leqslant 0.08$, on a

$$
\frac{1}{\varrho \log 2} \geqslant 17
$$

et

(20) $g(\varrho) \leqslant \frac{\log \log \left(k^{\prime}+1\right)+\left(1 / \log ^{2}\left(k^{\prime}+1\right)\right)-0.1159}{\log 4}-\frac{\varrho}{2}\left(\frac{k^{\prime}+1}{\log \left(k^{\prime}+1\right)}-1\right)$.

Avec le résultat de M. Waldschmidt, $x=2^{49} \log 3,(19)$, (6) et (20) donnent:

$$
\gamma=4.256 \cdot 10^{-16}, \quad k^{\prime}=3.3896 \cdot 10^{15}, \quad g(\gamma) \leqslant 2.48 .
$$

Avec le résultat de G. Rhin, $x=14$, (19) et (6) donnent:

$$
\gamma=0.0175, \quad k^{\prime}=81
$$

et un calcul direct donne

$$
g(\gamma) \leqslant 0.82
$$

Ceci démontre (i). Démontrons maintenant (ii). Il nous faut modifier pour cela la démonstration du théorème 1 de [9]: Avec les mêmes notations, on définit $M_{h}$ pour $-H \leqslant h \leqslant H$. On a:

$$
\delta_{h}=\log \frac{d\left(M_{h}\right)}{d(N)}=\{h \theta\} \log 2 .
$$

Soit $\left(u_{j} / v_{j}\right)$ le $j^{\text {ime }}$ convergent de $\theta$. On choisit $H=v_{j}$. Par le lemme des 3 distances, deux valeurs consécutives $\delta_{h}$ et $\delta_{h^{\prime}}$ vérifieront:

$$
\frac{1}{\log 2}\left|\delta_{h}-\delta_{h^{\prime}}\right| \leqslant\left\|v_{j-1} \theta\right\|<\frac{1}{v_{j}}=\frac{1}{H}
$$

A condition de choisir $H<x_{2}^{\tau}=x^{\theta \tau}$, on aura, si $M$ est hautement composé:

$$
\text { bén } M \lessgtr \frac{H \log 2}{x^{\theta(1-\tau)} \log x}+\frac{1}{H \log 2} \text {. }
$$

Pour chaque valeur de $j$, on pose:

$$
w=w_{j}=\left(v_{j}\right)^{2 /(\theta(1-\tau))} .
$$

On choisit $\lambda_{1}=1.3 ; \lambda_{2}=0.53 ; X=\exp \left(w^{\lambda_{1}}\right) ; X^{\prime}=\exp (w) ; X^{\prime \prime}=\exp \left(w^{\lambda_{2}}\right)$. On a:

$$
Q(X)=Q(X)-Q\left(X^{\prime}\right)+Q\left(X^{\prime}\right)-Q\left(X^{\prime \prime}\right)+Q\left(X^{\prime \prime}\right) .
$$

D'après (i) on $\mathrm{a}$

$$
Q\left(X^{\prime \prime}\right) \leqslant\left(\log X^{\prime \prime}\right)^{c_{1}+o(1)} \leqslant(\log X)^{0.41 c_{1}+o(1)} .
$$

Soit $N$ un nombre h.c.s. entre $X$ et $X^{\prime}, \varepsilon$ associé à $N$ et $x=2^{1 / e}$. On a $x$ $\sim \log N$ et donc

$$
w^{1+o(1)} \leqslant x \leqslant w^{\lambda_{1}+o(1)}
$$


Comme $H=v_{j}=(w)^{\theta(1-\tau) / 2}$, on a:

$$
x^{\theta(1-\tau) / 2+o(1)} \leqslant H \leqslant x^{\theta(1-\tau) /\left(2 \lambda_{1}\right)+o(1)}
$$

et la formule (21) donne

$$
\text { bén } M \leqslant x^{-\varrho_{1}} \quad \text { avec } \quad \varrho_{1}=\frac{\theta(1-\tau)}{2 \lambda_{1}}+o(1) .
$$

Comme il y a au plus $\log X$ nombres h.c.s. entre $X^{\prime}$ et $X$, la proposition 1 nous dit que:

$$
Q(X)-Q\left(X^{\prime}\right) \leqslant(\log X)^{1+g\left(Q_{1}\right)+o(1)} .
$$

Soit $N$ un nombre h.c.s. entre $X^{\prime \prime}$ et $X^{\prime}$; par un raisonnement similaire, en tenant compte de la décroissance de la fonction $g$, on obtient

$$
Q\left(X^{\prime}\right)-Q\left(X^{\prime \prime}\right) \leqslant\left(\log X^{\prime}\right)^{1+g\left(\varrho_{2}\right)+o(1)}=(\log X)^{\left(1+g\left(\varrho_{2}\right)\right) / \lambda_{1}+o(1)}
$$

avec $\varrho_{2}=\left(2-1 / \lambda_{2}\right) \frac{\theta(1-\tau)}{2} \geqslant 0.0148$

On a finalement:

$$
c_{2} \leqslant \max \left(0.41 c_{1}, 1+g\left(\varrho_{1}\right),\left(1+g\left(\varrho_{2}\right)\right) / \lambda_{1}\right) \leqslant 1.44 .
$$

Le raisonnement précédent n'a pas été optimisé complètement. La limite de la méthode est:

$$
c_{2}=1+g\left(\frac{\theta(1-\tau)}{2}\right)=1.37 .
$$

6. Démonstration du théorème 2. (i) Comme il a été remarqué dans [10], si $N$ est le nombre h.c.s. immédiatement avant $n_{j}^{\prime}$, on a

$$
\text { bén } n_{j}^{\prime} \ll x^{-\gamma} \text {. }
$$

Si $N^{\prime}$ est le nombre h.c.s. suivant $N$ (on sait que $d\left(N^{\prime}\right) \leqslant 2 d(N)$ ) si $n_{j+1}^{\prime}<N^{\prime}$ on a également bén $n_{j+1}^{\prime} \leqslant x^{-\gamma}$ et la proposition 3 permet de conclure. Si l'on a $n_{j}^{\prime}<N^{\prime}=n_{j+1}^{\prime}$, la même démonstration de la proposition 3 montre que $N^{\prime} / n_{\mathbf{k}}^{\prime}$ ne peut pas être trop petit. On peut finalement prendre

$$
\alpha_{1}<1-\gamma
$$

(ii) La démonstration de (i) marche aussi et donne le résultat pour $\alpha_{2}<1-\gamma$. On peut faire un peu mieux en observant que dans un nombre hautement composé, la suite des exposants des nombres premiers successifs est décroissante. Avec les notations du paragraphe 4, on a donc toujours $\mu_{k}^{\prime}\left(n_{j}\right) \mu_{k}^{\prime \prime}\left(n_{j}\right)=0$, et donc

$$
\left|\mu_{k}^{\prime}+\mu_{k}^{\prime \prime}\right|=\left|\mu_{k}^{\prime}-\mu_{k}^{\prime \prime}\right|=\left|\mu_{k}\right| .
$$

En faisant dans la démonstration de la proposition $3 M=n_{j}, M^{\prime}=n_{j+1}$, on obtient

$$
t_{k} \leqslant\left|\mu_{k}\left(n_{j}\right)\right|+\left|\mu_{k}\left(n_{j+1}\right)\right|
$$

et par (16), on voit que l'on peut choisir n'importe quel $\alpha_{2}$ vérifiant $\alpha_{2}<(\theta-\gamma) / 2$.

(iii) Cela résulte de (18) et de la proposition 2. On peut choisir $\alpha_{3}<(\theta-\gamma) / 2$.

(iv) Soit $n_{j}<n<n_{j+1}$. On sait (cf. [3] et [9]) que

$$
\frac{n_{j+1}}{n_{j}} \leqslant 1+\frac{1}{\left(\log n_{j}\right)^{0.1}}
$$

Avec la définition du bénéfice (5), on a:

$$
0 \leqslant \varepsilon \log \frac{n}{n_{j}}=\log \frac{d(n)}{d\left(n_{j}\right)}+\text { bén } n-\text { bén } n_{j} \leqslant \varepsilon \log \frac{n_{j+1}}{n_{j}} \leqslant \frac{\varepsilon}{\left(\log n_{j}\right)^{0.1}} .
$$

Supposons d'abord que bén $n \geqslant x^{-\varrho}$ avec $0<\varrho<\gamma$. La relation précédente donne, puisque bén $n_{j} \ll x^{-\gamma}$ et que $y<0.1$ :

$$
\log \left(d(n) / d\left(n_{j}\right)\right) \leqslant-x^{-e / 2} .
$$

Ce qui entraîne le résultat pour tout $\alpha_{4}<1$. Supposons maintenant que bén $n \leqslant x^{-Q}$, alors la proposition 3 nous donne le résultat pour tout $\alpha_{4}<1-\varrho$, c'est-à-dire pour tout $\alpha_{4}<1-\gamma$.

7. Démonstration du corollaire 1. Observons d'abord que, pour $n_{j}$ hautement composé, on a:

$$
d\left(n_{j}\right) \geqslant 2^{\left(\log \left(n_{j}\right) / \log \log \left(n_{j}\right)\right) /(1+o(1))} .
$$

En effet, Ramanujan a démontré une telle formule pour les nombres h.c.s. (cf. [14], p. 118), et si $N$ et $N^{\prime}$ sont les deux nombres h.c.s. encadrant $n_{j}$, on a:

$$
d\left(n_{j}\right) \geqslant d(N) \geqslant \frac{1}{2} d\left(N^{\prime}\right) \geqslant 2^{\left(\log N^{\prime} / \log \log N^{\prime}\right)(1+o(1))} \geqslant 2^{\left(\log n_{j} / \log \log n_{j}\right)(1+o(1))}
$$

Ensuite, on a de façon évidente:

$$
n \text { largement composé } \Rightarrow n \text { est }(d+f) \text { h.c. }
$$

Il suffit de montrer que si $n$ n'est pas largement composé, $n_{j}<n<n_{j+1}$, alors

$$
d(n)+f(n)<d\left(n_{j}\right)
$$

Or d'après (iv) du théorème 2,

$$
d(n) \leqslant d\left(n_{j}\right)-\frac{d\left(n_{j}\right)}{\exp \left(\left(\log n_{j}\right)^{\alpha_{4}}\right)}
$$

et la condition (3) et l'équivalence $n_{j} \sim n_{j+1}$ achèvent la démonstration. 
De façon évidente, on a:

$$
n \text { h.c. } \Rightarrow n \text { est }(d-f) \text { h.c. }
$$

Il suffit de montrer que la fonction $(d-f)$ est croissante sur les nombres h.c., ce qui se fait en utilisant le théorème 2, (ii).

8. Démonstration du corollaire 2. Soit $N$ un nombre hautement composé supérieur associé à $\varepsilon$, et $x=2^{1 / \varepsilon}$. Chaque nombre premier $p \leqslant x$ ne divise pas soit $n$ soit $n+1$. Donc la formule (10) donne:

$$
\begin{aligned}
\text { bén } n+\text { bén }(n+1) & \geqslant \sum_{p \leqslant x}-\varepsilon a_{p} \log p+\log \left(a_{p}+1\right) \\
& \geqslant \sum_{p \leqslant x}-\varepsilon \log p+\log 2=\sum_{p \leqslant x} \varepsilon \log (x / p)
\end{aligned}
$$

et donc:

$$
\text { bén } n+\text { bén }(n+1) \gtrsim((\log 2) x) / \log ^{2} x \text {. }
$$

Nous remarquons ensuite que par (5)

$$
\log \frac{d(n)}{d(N)}=\varepsilon \log \frac{n}{N}-\text { bén } n .
$$

Si, $N \leqslant n<N^{\prime}$ où $N$ et $N^{\prime}$ sont deux nombres h.c.s. consécutifs, $N^{\prime} / N \leqslant x$ et

$$
\log \frac{d(n)}{d(N)} \leqslant- \text { bén } n+(1+o(1)) \log 2 \text {. }
$$

Supposons que ni $n$ ni $(n+1)$ ne sont largement composés. Soit $n_{j}$ le nombre h.c. précédant $n$ et $N$ le nombre h.c.s. précédant $n_{j}$. Ou bien on a:

$$
\text { bén } n \geqslant 3 \log 2 \text { et bén }(n+1) \geqslant 3 \log 2
$$

et alors (23) montre que $d(n)<\frac{1}{2} d(N), d(n+1)<\frac{1}{2} d(N)$ et donc

$$
d(n)+d(n+1)<d(N)+d(N+1) .
$$

Ou bien l'un des deux nombres $n$ ou $(n+1)$, par exemple $n$, vérifie bén $n<3 \log 2$, et l'on a par (23) et $(22)$;

$$
\begin{aligned}
& \text { bén }(n+1) \geqslant \frac{x \log 2}{\log ^{2} x}(1+o(1)), \\
& d(n+1) \leqslant d(N) 2^{\left(-x / \log ^{2} x\right)(1+o(1)) .}
\end{aligned}
$$

En appliquant (iv) du théorème 2 , on $\mathrm{a}$ :

$$
d(n) \leqslant d\left(n_{k}\right)-\frac{d\left(n_{k}\right)}{\exp \left(\log n_{k}\right)^{\alpha_{4}}}
$$

et comme $d(N) \leqslant d\left(n_{k}\right)$ et que $x \sim \log N \sim \log n_{k}$, on a

$$
d(n)+d(n+1)<d\left(n_{k}\right)<d\left(n_{k}\right)+d\left(n_{k}+1\right) .
$$

On démontrerait de même que si $n+1$ est hautement composé, alors pour tout $m<n$, on a:

$$
d(m)+d(m+1)<d(n+1)
$$

en utilisant (iii) du théorème 2 .

9. Quelques problems ouverts. Les résultats précédents sont basés sur les approximations diophantiennes de

$$
\theta=\frac{\log (3 / 2)}{\log 2}=\frac{\log \left(d\left(p^{2}\right) / d(p)\right)}{\log d(p)}
$$

Que se passe-t-il si on remplace la fonction $d$ par une fonction multiplicative $\delta$, telle que $\delta\left(p^{\alpha}\right)$ dépende seulement de $\alpha$ et soit voisin de $\alpha+1$ mais tel que

$$
\frac{\log \left(\delta\left(p^{2}\right) / \delta(p)\right)}{\log \delta(p)}
$$

soit un nombre de Liouville? Peut-on construire une telle fonction avec une quantité de nombres $\delta$-h.c. qui dépasse toute puissance de $\log x$ ? (cf. [19]).

Il n'y a pas non plus de bonne majoration pour le nombre de nombres hautement abondants, superabondants (cf. [4]) où pour les "sparsely totient numbers" inférieurs à $X$ (cf. [6]).

Peut-on améliorer le théorème 2 et montrer qu'il existe $\beta$ tel que:

$$
\frac{d\left(n_{j+1}\right)}{d\left(n_{j}\right)} \leqslant 1+\frac{1}{\left(\log n_{j}\right)^{\beta}} ?
$$

Par contre je conjecture que

$$
\frac{n_{j+1}}{n_{j}} \leqslant 1+\frac{1}{\left(\log n_{j}\right)^{\beta}}, \quad j \geqslant j_{0}
$$

est faux, pour tout $\beta>0$, et ceci malgré la proposition 4 qui ne marche que parce que $N$ est h.c.s.

Enfin, existe-t-il une infinité de nombres $n$ non largement composés et vérifiant $m<n \Rightarrow m d(m)<n d(n)$ ?

A jouté à la lecture des épreuves:

G. Rhin vient d'obtenir dans le temme 2 la valeur de $x=7.616$ (Approximants de Padé et mesure d'irrationalité, Sém. Th. Nombres, Paris 1985-86, à paraitre chez Birkhaüser, Progress in Mathematics). Cela donne, dans le théorème $1, c_{1} \leqslant 1.71$.

C. J. Mozzochi vient d'obtenir dans (1) la valeur de $\tau=\frac{11}{20}-\frac{1}{384}=0.547396$ (On the difference between consecutive primes, J. Number Theory 24 (1986), p. 181-187). 
[1] L. Alaoglu and P. Erdôs, On highly composite and similar numbers, Trans. Amer. Math. Soc. 56 (1944), p. 448-469.

[2] G. Bessi et J. L. Nicolas, Nombres 2-hautement composés, J. Math. pures et appl. 56 (1977), p. 307-326.

[3] P. Erdôs, On highly composite numbers, J. London Math. Soc. 19 (1944), p. 130-133,

[4] P. Erdős et J. L. Nicolas, Répartition des nombres superabondants, Bull. Soc. Math. France 103 (1975), p. 65-90.

[5] D. R. Heath-Brown and $\mathrm{H}$. Iw aniec, On the difference between consecutive primes, Inv. Math. 55 (1979), p. 49-69.

[6] D. W. Masser and P. Shiu, On sparsely totient numbers, Pacific J. Math. 121 (1986), p. $407-426$.

[7] H. L. Montgomery and R. C. Vaughan, The large sieve, Mathematika 20 (1973), p. 119-134.

[8] J. L. Nicolas, Ordre maximal d'un élément du groupe des permutations et highly composite numbers, Bull. Soc. Math. France 97 (1969), p. 129-191.

[9] - Répartition des nombres hautement composés de Ramanujan, Canad. J. Math. 23 (1971) p. $116-130$.

[10] - Répartition des nombres largement composês, Acta Arith. 34 (1980), p. 379-390.

[11] - Sur les entiers $n$ pour lesquels il y a beaucoup de groupes abéliens dordre $n$, Annales Inst. Fourier 28 (1978), p. 1-16.

[12] S. Pillaî, Highly abundant number, Bull. Calcutta math. Soc. 35 (1943), p. 141-156.

[13] - Highly composite numbers of the th order, J. Indian Math. Soc. 8 (1944), p. 61-74.

[14] S. Ramanujan, Collected papers, University Press, Cambridge 1927.

[15] G. Robin, Méthodes d'optimisation pour un problème de théorie des nombres, R.A.I.R.O. Informatique théorique 17 (1983), p. 239-247.

[16] - Grandes valeurs de la fonction somme des diviseurs et hypothèse de Riemann, J. Math. pures et appl. 63 (1984), p. 187-213.

[17] J. B. Rosser and L. Schoenfeld, Approximate formulas for some functions of prime numbers, Illinois J. Math. 6 (1962), p. 64-94.

[18] N. Slater, Gaps and steps for the sequence $n \theta \bmod 1$, Proc. Cambridge Phil. Soc. 63 (1967), p. 1115-1123.

[19] T. H. Tran, Nombres hautement composés de Ramanujan généralisés, C. R. Acad. Sci. Paris, Ser. A-B, 282 (1976), p. 939-942.

[20] M. Waldschmidt, $A$ lower bound for linear forms in logarithms, Acta Arith, 37 (1980), p. $257-283$.

DÉPARTEMENT DE MATHÉMATIQUES

UNIVERSITE DE LIMOGES

123 Avenue A. Thomas

France

Reçu le 13.6.1986

$(1650)$

\section{Simultaneous diophantine approximation and $I P$-sets}

by

\section{H. Furstenberg and B. Weiss (Jerusalem)}

Introduction. Weyl's theorem on equidistribution, which superseded earlier results by Hardy and Littlewood, implies that for any real polynomial $p(t)$, and $\varepsilon>0$, the diophantine inequality

$$
|p(x)-p(0)-y|<\varepsilon, \quad x \neq 0,
$$

has a solution ([9]). A multidimensional version ([7]) tells us that we can solve

$$
\left|p_{j}(x)-p_{j}(0)-y_{j}\right|<\varepsilon, \quad j=1,2, \ldots, J, \quad x \neq 0
$$

simultaneously for any finite set of real polynomials $\left\{p_{j}(x)\right\}$.

In terms of the exponentials

$$
\varphi_{j}(n)=\exp \left(2 \pi i p_{j}(n)\right)
$$

the foregoing states that the functions $\varphi_{j}$ on the integers return simultaneously arbitrarily close to their values at 0 .

We shall present a general principle here according to which certain functions on $Z$ "recur", and the recurrence takes place along specified sets of integers, the IP-sets which we shall presently define. Because of the information on the sets of recurrence, it will follow that the functions in this class recur simultaneously. Each combination of such functions presents us with a result on diophantine approximation. Our principal result will be that if $p_{1}\left(t_{1}\right), p_{2}\left(t_{1}, t_{2}\right), \ldots, p_{l}\left(t_{1}, t_{2}, \ldots, t_{l}\right)$ are arbitrary real polynomials vanishing for $t_{i}=0$, then for any $\varepsilon>0$, the system of inequalities

$$
\begin{array}{r}
\left|p_{1}\left(x_{1}\right)-x_{2}\right|<\varepsilon, \\
\left|p_{2}\left(x_{1}, x_{2}\right)-x_{3}\right|<\varepsilon, \\
\ldots \ldots \ldots \\
\left.\ldots \ldots x_{l}\right)-x_{l+1} \mid<\varepsilon
\end{array}
$$

has a solution in non-zero integers.

IP-sets are closely tied up with the notion of recurrence in topological dynamics. For the background in this we refer the reader to [2], [3] and [4]. 